\title{
LONG CORRIDOR SURVEY FOR HIGH VOLTAGE POWER LINES DESIGN USING UAV
}

\author{
D. Skarlatos ${ }^{\text {a }}$, V. Vamvakousis ${ }^{\text {a }}$ \\ ${ }^{a}$ Cyprus University of Technology, Civil Engineering \& Geomatics Department, 30 Archbishop Kyprianou, Limassol, 3036, Cyprus \\ - (dimitrios.skarlatos, v.vamvakousis)@ cut.ac.cy
}

\section{Commission II}

KEY WORDS: UAV flight, DSM, corridor survey, mapping, civil engineering, power lines design, low cost

\begin{abstract}
:
The term Unmanned Aerial Vehicle (UAV) is often directly associated with the armed forces due to their widely-criticized use of such vehicles on the modern battlefield. However, with the advancement of UAV technology, the acquisition and operational cost of small civilian UAV have reduced while their functionalities have increased. Therefore, a wide variety of new civilian applications have emerged. Mapping industry has been benefited as affordable UAV can partially replace traditional platforms, such as helicopters and small aircrafts, for low altitude photography acquisition. Although relatively new to the industry, the use of UAV is rapidly commercialized and they are expected to have a sizeable impact on the mapping industry in the coming years. The aim of this work was to test the use of a low-cost UAV for orthophoto production and Digital Surface Model (DSM) creation, to be used for the design of a new $23 \mathrm{~km}$ high voltage line of Electricity Authority of Cyprus.
\end{abstract}

\section{INTRODUCTION}

\subsection{UAV emerging applications}

Since 2010, researchers on various disciplines have been showing growing interest in utilizing Autonomous Unmanned Aircraft Vehicles (AUAV) for diverse non-military purposes. Currently, the main AUAV revenue is defense related. However, several market studies (Zhang et al., 2011) indicate that the worldwide UAV market will expand significantly in the next decade. The only drawback seems to be the lack of operational rules (Eisenbeiß, 2009 and Saari et al., 2011), but one by one, most countries have established rules and regulations for AUAV. Applications of AUAVs both fixed wing and multirotor, were initially considered for dirty, dull and dangerous applications. For instance, the use of UAVs in 'dirty' situations, such as radioactive contamination, was documented after the Fukushima reactor accident (Saari et al., 2011; Rango et al., 2006). The use of UAVs for 'dull' operations includes their use in frontier surveillance (Semsch, et al., 2009; Kontitsis, et al., 2004). Several simpler civilian applications have emerged also, such as forest and agricultural applications (Remondino et al., 2011; Kyratzis, et al., 2017), autonomous surveillance (Kontitsis et al., 2004; Srinivasan et al., 2004), emergency and disaster management (Ameri at al., 2008), traffic surveillance and management (Heintz et al., 2007), photogrammetry and 3D modeling (Remondino et al., 2011; Skarlatos et al., 2013), etc.

The reduced cost of these platforms has placed AUAV as a viable substitute to current aerial platforms, such as full-scale manned aircraft. Main advantages are reduced acquisition and maintenance cost, fast deployment, endurance, versatility, many different implementations, high resolution. Their recent widespread adoption was based on their enhanced autonomous capability, supported by Global Positioning Systems (GPS) and
Inertial Navigation Systems (INS). Some AUAV are fully capable to perform flights autonomously, from take-off to landing. Since 1960s, only few private mapping companies, have adopted UAVs in their production line. The new ability of UAVs to flight autonomously, on exact predefined paths, limiting user intervention to forward and side overlaps selection, was the main reason that AUAV are now considered standard geomatic equipment such as GPS and theodolites, sometimes at the fraction of the cost of the latter. Numerous projects are being reported emphasizing the need for small and medium area on-demand timely mapping. In fact, AUAV have successfully filled in the gap in aerial platforms between tripods and low altitude balloons to light manned aircrafts. Nevertheless, the threshold in area coverage where AUAV are cost efficient in comparison to traditional manned aircrafts is not apparent. The cost function depends on the equipment on board as well as the type of platform. The end user, ordering the final products, is rarely concerned with such technicalities.

This paper describes the planning, execution and processing of a $23 \mathrm{~km}$ corridor survey of $250 \mathrm{~m}$ width, on average, on behalf of the Electricity Authority of Cyprus (EAC). The corridor survey was meant to be used for planning and design a new highvoltage line, to join the electrical substations of Alhambra and Kofinou. The region of interest was covered by high hills, dead areas and forest. Land survey using RTK GPS were usually engaged by EAC for such surveys, mainly because of the available personnel and the lack of photogrammetric experience. Due to the length of this survey, the lack of quantitative and qualitative information gathered during previous land surveys, EAC wanted to investigate photogrammetric processing as an alternative. The cost of hiring traditional manned aircraft for the task was out of budget, since no photogrammetric airplanes are stationed in Cyprus. Use of AUAV data acquisition seemed an interesting and affordable alternative photogrammetric solution.

* Corresponding author 
Nevertheless, covering such a large corridor using fixed wing AUAV proved to be far from a trivial task, both in terms of flight execution, ground control point measurement and data processing. All these problems, along with solutions and accuracies are being discussed in the paper, along with the cost estimation.

\subsection{Previous Work}

According to the Unmanned Vehicle System (UVS) International definition, a UAV is a generic aircraft design to operate with no human pilot onboard (Remondino et al., 2011). Even though there is no pilot physically present in the aircraft, this doesn't mean that it can also fly autonomously. In many cases, the crew responsible for a UAV is larger than that of a conventional aircraft. The aircraft is controlled from the ground (the Ground Control Station or GCS), so it needs reliable communication links to and from the aircraft, but also to the local Air Traffic Control (ATC) authorities if required.

Technological advances have significantly changed land surveying profession, in the last two decades. Technology improvements in acquisition of topographic data has enabled huge volumes of data gathered in short time, while final products are of higher quality. Following that, end-users now require more, better and affordable three-dimensional spatial information to be delivered faster. Besides laser scanning, photogrammetry is also gaining more and more attention every year, especially after implementing complex algorithms from computer vision in software solutions, and introduction of unmanned aerial vehicles AUAV as an improved photogrammetric platform.

Due to the low operation altitude, UAVs deliver high resolution photos in terms of ground sampling distance and can therefore compete with airborne large format digital camera systems (Skarlatos et al., 2015), which in return may cover larger areas with a single photo, hence reducing control points, while maintaining high accuracy due to pre calibration Major advantage of using UAVs is also the cost factor, as UAVs are less expensive and have lower operating costs than manned aircrafts. The main disadvantage of UAVs is their payload limitations. These limitations affect the sensors, both cameras and navigation systems.

The interest in UAV for civilian applications has rapidly grown in last few years due to emergence of sophisticated hardware and software systems/algorithms to support semi-autonomous or fully autonomous control.

\section{METHODOLOGY OF INITIAL PLANNING}

In 2014 Electricity Authority of Cyprus was planning the construction of a new high voltage line, between Alampra and Kofinou power distribution centers (Fig. 1). The established practice within the organization was the use of land surveyors covering the corridor area with RTK GPS receivers. The final output of such surveys is a vector plot with man-made constructions, roads and contours. Man-made features are rare in those remote areas, where the high voltage power lines are to be constructed. Given the roughness of the ground the surveyors are collecting points is a sparse grid of approximately $30 \mathrm{~m}$ and they try to collect points along break lines in order to fully describe terrain. The field work progress is on average $1.7 \mathrm{~km}$ for a crew of two surveyors with GPS, per day. The final vector plot has only limited information regarding the state of the features (roads, houses other man made features etc), existing vegetation, height of trees and cultivated areas. At the same time the low density Digital Surface Model (DSM) is sparse to fully describe the terrain. Tree canopy information is missing, despite the fact that it is critical to the project, as long as EAC needs to know the clearance between hanging power cables and underlying canopy. Therefore, the qualitative information of the land survey is poor for the study and the acquisition process slow, tedious and rather expensive in terms of human resources. Because of the aforementioned limitations and shortcomings of ground surveys, EAC considered using UAVs to perform the corridor survey, hoping for:

- Fast data acquisition

- Better and more detailed terrain modelling, including tree canopy

- Generation of orthophoto mosaic with much more detail than the vector plot, hence superior site awareness

- Aerial photographs could be used in a court of law as evidence of the situation before the power lines were constructed, in case of extremely high compensations demanded by land owners

- Faster delivery of final product

- Cost reduction, with a relatively small investment in a new UAV.

\subsection{Study Area}

The study area (Fig. 1) is forested mountainous area with intense natural terrain and natural coverage and dead areas. The original request was for a $100 \mathrm{~m}$ corridor width, but the captured one was on average $250 \mathrm{~m}$ wide and $23 \mathrm{~km}$ in length. Terrain altitude varies from 130 to $500 \mathrm{~m}$, with some steep slopes mainly in gorges.

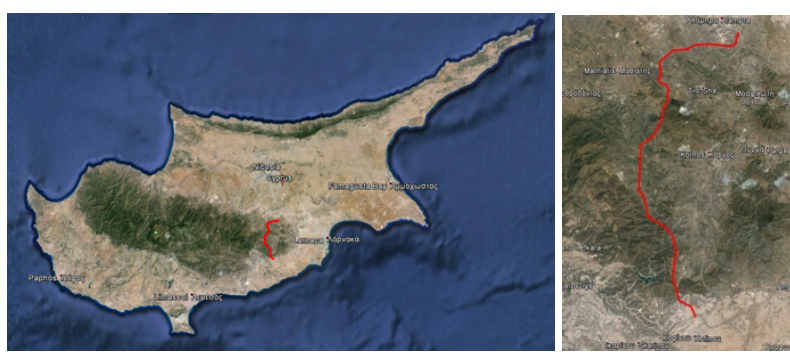

Figure 1. Study area

\subsection{Flights}

Fixed wing drones are preferred to multi rotor ones, when used to collect images over bigger area of interest, because of their high speed and energy efficiency. On the other side, they require more space for take-off and landing. Therefore, it was decided that a fixed wing UAV should be used in this project. In preparation of the flight planning, a request for flight permission was send to the department of Civil Aviation Authority (CAA) of Cyprus. It was envisaged to make the whole area in two flights, using the lab's long flight fixed wing AUAV, hence the initial request was asking for a no fly zone over the whole corridor and up to $400 \mathrm{~m}$ above ground, for half a day. The lack of a legal framework concerning the usage of AUAV at the time, obliged civil aviation authority to issue a strict permit based on regulations for RC models. The main restrictions were:

- Maintain eye contact with the aircraft at not more than 500 and throughout the flight. 
- The flights should not exceed a ceiling of 400 feet from the ground and kept a safe distance 150 meters from residential areas and 50 meters from humans, animals etc.

- An army officer should be present during the flight, to verify that photos taken during the flight, do not show military infrastructure.

Given the directions and restrictions set by the department of CAA, it was decided to use the first generation SwingletCAM from Sensefly, which weighs less than 500 gr, is composed of a light wingspan combined with a u-BLOX GPS chip, an attitude sensor, a radio transmitter and an autopilot circuit board. The on-board camera is an uncalibrated Canon IXUS 220IS, with $4.3 \mathrm{~mm}$ focal length over a $6.2 \times 4.6 \mathrm{~mm}$ CMOS $3000 \times 4000$ pixel sensor, with 1.55 microns physical pixel size. Power supply is a small Lithium-Ion $1350 \mathrm{mh}$ battery and reported flight autonomy is about $25 \mathrm{~min}$.

Flight planning proved a complicated process, both in terms of logistics as well as flight design. Given the instructions of the CAA for $500 \mathrm{~m}$ line of sight from ground station, the selection of available and accessible open areas for take-off and landing, through rough dirt roads was a challenging task. Regarding the flight line design, Swinglet's software didn't allow, terrain following mode, at that time. It only allowed flight height variations with reference the altitude at the take-off location. To maintain fixed scale to all photos, the terrain altitude variations were examined at Google Earth and the flight height of the AUAV were adjusted accordingly, leading to a different flying height per flight line. The maximum allowed flying height from the CAA was adopted, since at $130 \mathrm{~m}$, the expected ground pixel size of Swinglet's camera was $0.06 \mathrm{~m}$, which was enough to surpass EAC's planimetric accuracy specifications of $0.10 \mathrm{~m}$. It should be noted that the ground resolution was not correlated with the planimetric and vertical accuracy specifications, set by EAC.

Being a corridor survey, each leg has different azimuth to the previous one. Using the default software design (Fig. 2), which by default allows photos taken only when in straight line, the photo coverage among consecutive lines are weak and do not provide enough tie points for the bundle adjustment and DSM generation, leaving weak areas on the project. Instead, the flight lines were extended, so that there was enough overlap among photos for both bundle adjustment and DSM, at the expense of extra flying time.

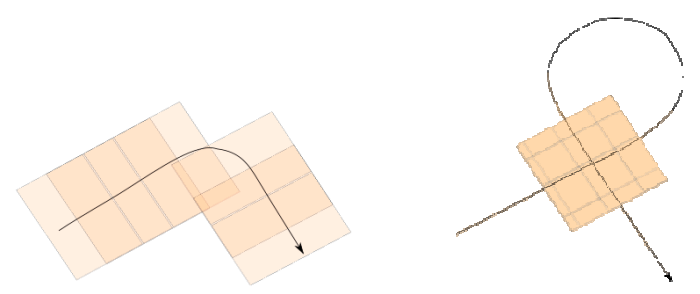

Figure 2. (a) On the left, the default flight line design, (b) on the right the modified version

Several flights covering the entire region were planned. Instead of making a single pass from the area and then coming straight back to the take-off position for landing, at each flight the AUAV was taking photos both ways going and coming back, ensuring double coverage of the area of interest. Hence, in total,
$53 \mathrm{~km}$ were covered by photos, with total coverage 8.61 square kilometers. Forward overlap was set to $80 \%$.

The flight campaign lasted 4 days, gathering 980 photos in total. Despite the meticulous flight planning, during the draft aerial triangulation in Agisoft's PhotoScan Professional ${ }^{\circledR}$, it was realized that there were two weak areas (paragraph 3.2), without enough tie points. To overcome the problem, two additional flights were planned and executed as area surveys with $70 \%$ $80 \%$ overlaps, adding an extra day, to the flight campaign. The total number of photos including the additional flights was 1293. The difference of coverage over the problematic areas is demonstrated among Fig. 3b and Fig. 9. Having completed the alignment, a draft Digital Surface Model (DSM) and an orthophotograph were created, to be used as guide for ground control point acquisition.

\subsection{Ground control points}

Ground control points (GCP), used in this project, were naturally points, collected after the flight, using Global Navigation Satellite System (GNSS). The selection of points was done over the draft orthophoto created in the previous phase. Use of pre-signalized GCPs was not considered as an option, because in a corridor survey, there is high risk that the edges might not be executed precisely enough, when dealing with a light UAV sensitive to wind gusts.

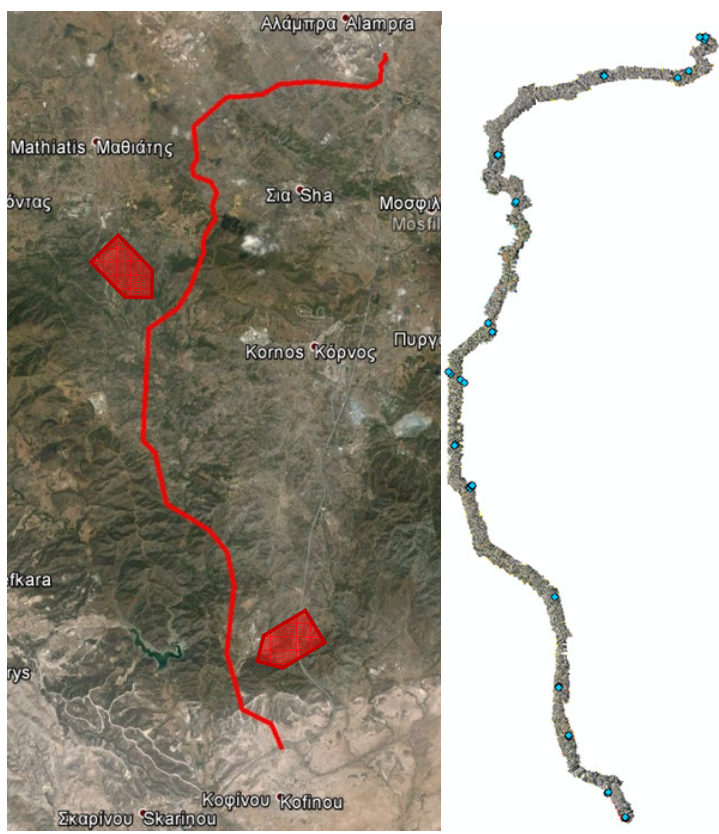

Figure 3. (a) on the left the design of the high voltage line, over google maps, with arrows showing problematic areas, (b) on the right the initial orthophoto using 980 photos and the positions of the 32 used GCPs

Both identification of natural points at the desired locations and planning to access these locations through rough dirt roads, was challenging. Sometimes the travelling time from point to point separated by $500 \mathrm{~m}$ in map, was almost one hour long, driving a $4 \times 4$ vehicle around a valley. Given the problems confronted it was decided to prioritize collection of GCP in accessible areas. Therefore, during the initial 3 days, 42 GCP were collected in 17 locations. At least two GCP were collected in each visited position, to be sure that the selected natural points were to be easily identifiable in the aerial photos. The maximum span 
between successive locations was $3800 \mathrm{~m}$ long, hence significant residuals in $\mathrm{Z}$ were expected.

Out of the 42 GCPs, 32 were used for the initial processing of the block. Some GCPs could not be identified in the photos and those with big residuals were also excluded from processing, as they might have been moved between the photo and GCP acquisition. The use of this reduced set of GCPs, proved inadequate for the accuracy specifications of the project, as tested over the final product. Therefore, a new field campaign was planned to collect additional GCPs, every $325 \mathrm{~m}$, with maximum spam of $500 \mathrm{~m}$. At the end of this additional 6-day campaign 189 GCPs were collected in total.

\section{DATA PROCESSING}

Photogrammetric processing was conducted in Agisoft's PhotoScan Professional ${ }^{\circledR}$, which employs Structure from Motion (SfM) to automatically estimate both camera parameters (camera self-calibration) and spatial positioning of the photos. After the aerial triangulation completion (optimization), Multi View Stereopsis (MVS) is used to produce a very dense point cloud, from which DSM is created using Triangulated Irregular Network (TIN). Usually when processing aerial photography, the 'height field' option is used to create a traditional $2.5 \mathrm{D}$ DSM, to be further used for orthophoto creation. Although this seems a trivial and highly automated process, when the project was performed, three problems were encountered.

\subsection{Initial approximations}

During the alignment phase, the user may import approximate projection center positions (photo centers) recorded by the onboard navigation system, as initial approximation for the aerial triangulation. This option provides information to restrict matching of features across images, which not only significantly reduces SfM time in corridor surveys, but also increases the chances for a successful orientation. Given the number of images and the corridor layout, such option would have reduced processing time and provide better results. The SwingletCAM UAV can record both positions and rotations, on the ground computer, rather than on the on-board pilot. Hence, when there was no connection among ground computer and UAV, this information was missing. Due to rough terrain, approximately $20 \%$ of the positions were not recorded.

To overcome this problem and gain control over the orientation process and minimize blunders, the area was divided in 9 sublocks, by merging full flights together. The blocks were aligned using the option of initial approximations, where the photos with missing data were aligned to those with initial approximations. The orientation was then exported to be used as initial approximation to the integrated block. At the final solution, all 1293 photos had initial approximation values, which speeded up process and avoided erroneous stitching. At the same time, it also facilitated control point measurement since the GCP were close to the positions suggested by the software.

\subsection{Weak areas}

As previously mentioned, there were two instances where the software could not connect the photos by automatically extracted tie points. After examining the photos over these areas, the problem was attributed to the combination of intense slopes and low flying height, causing significant viewing angle changes from image to image across ridges and minimize overlaps (Fig. 4). This problem, once recognized was easily solved by executing additional flights over these areas, in block layout, with large overlaps. When the new photos were added in the block, the alignment issues were solved.

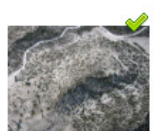

IMG_3213.JPG

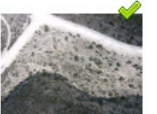

IMG_3218.JPG

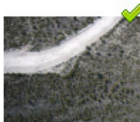

IMG_3223.JPG

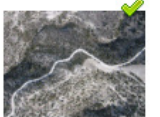

IMG_3214.JPG

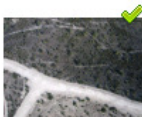

IMG_3219.JPG

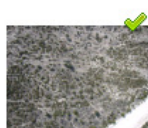

IMG_3224.JPG

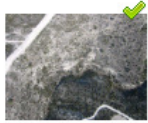

IMG_3215.JPG

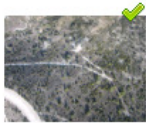

IMG_3220.JPG

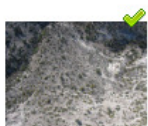

IMG_3225.JPG

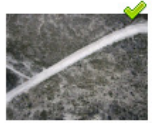

IMG_3216.JPG

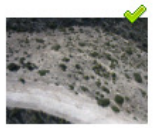

IMG_3221.JPG

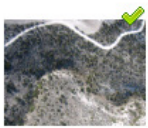

IMG_3226.JPG

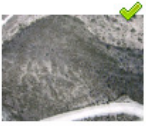

IMG_3217.JPG

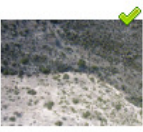

IMG_3222.JPG

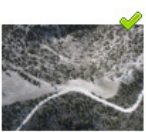

IMG_3227.JPG
Figure 4. Consecutive photos above a problematic area. It is apparent there are not enough overlaps, nor the slopes look alike from photo to photo.

\subsection{Alignment errors}

The two preceding problems were easy to recognize and solve. The alignment errors were revealed during the DSM inspection phase, as linear features of steep ridges with varying heights of up to $4 \mathrm{~m}$ in height (Fig. 5), in otherwise flat areas.

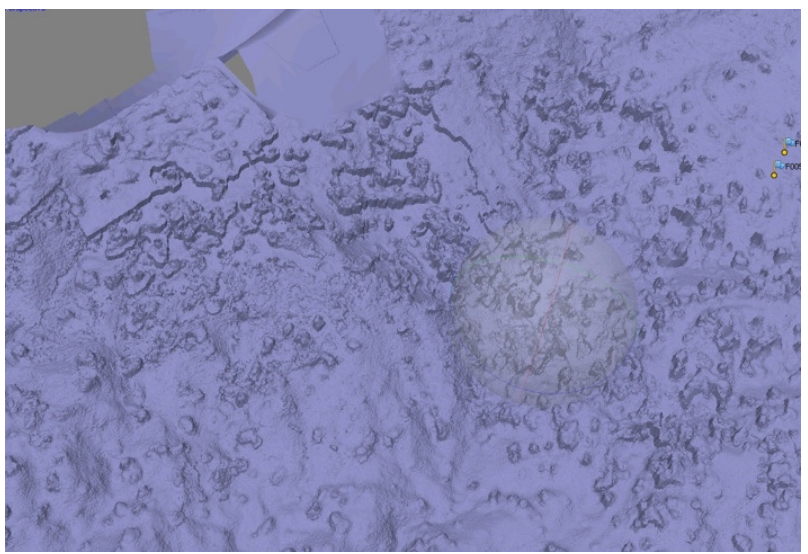

Figure 5. Detail of steep ridges, on the top of the image.

Such errors can be attributed to erroneous orientation (otherwise bundle adjustment, or optimization according to PhotoScan terminology). During the alignment phase, over two million tie points were produced. Among these automatically created tie points, there are several visually identifiable blunders, which rest outside the main concertation of tie points. Including these blunders in the solution would affect both the optimization and final products. These blunders were manually selected and deleted, before proceeding (Fig. 6). Still, there will be several blunders remaining in the main concentration of the tie points. These blunders were removed through a manual robust regression process. During this empirical recursive process, the user sets loose thresholds to select and erase possible blunder tie points with excessive 'reprojection error', 'reconstruction uncertainty' and 'projection accuracy'. After the optimization, the user tightens the thresholds and repeats the 
optimization, until these errors become low and in accordance to project specifics. Caution must be exerted, so that in each iteration, no more than $10 \%$ of the points are to be eliminated and the finally remaining points are enough for block bundle adjustment.

Alignment
- Manual elimination of blunders based
on visual inspection

Figure 6. Processing diagram

The selection of camera parameters to be optimized, is the last factor to be considered. However, this also affects the final solution. Over parametrization of the camera, while there is not enough information to reliably estimate the parameters may have negative effects to the final solution. Having gone by the process of eliminating blunder tie points, it was expected that the optimization and subsequent final results, such as DSM and orthophoto would be correct.

Nevertheless, since Agisoft PhotoScan is a versatile software able to accommodate either aerial or terrestrial photos, default parameters are rarely set correctly for each project/case. As an example, the default settings for marker (GCP) accuracy is set to $0.005 \mathrm{~m}$. This value does not represent the accuracy of GCP on this case, where GCP were collected using GNSS, with an expected accuracy of $0.02 \mathrm{~m}$ in XY and $0.03 \mathrm{~m}$ in Z. Keeping the default parameters during optimization, means that the GCPs will influence the solution much more than they should. Therefore, all parameters should be set with reasonable values. In this case, the settings used can be seen in Table 7.

After setting properly the parameters, optimizing and recreating DSM and orthophoto, the aforementioned alignment errors disappeared, highlighting the need to correctly set all parameters and not to rely on the default settings.

\begin{tabular}{|l|c|}
\hline Parameter & Setting \\
\hline Camera accuracy [m] & 20 \\
Camera accuracy [deg] & 4 \\
Marker accuracy [m] & 0.03 \\
Market accuracy [pix] & 1 \\
Tie point accuracy [pix] & 1 \\
Camera parameters & f,cx,cy,k1,k2, \\
& k3,p1,p2 \\
\hline
\end{tabular}

Table 7. Settings used for bundle adjustment (optimization)

\subsection{Final results and accuracy evaluation}

After overcoming the reported adjustment issues, final optimization using the initial $32 \mathrm{GCPs}$, reported 2.05 pixel reprojection error in tie points, 1.07 pixel reprojection error in GCP and X, Y, Z errors of $0.07,0.09,0.14 \mathrm{~m}$ respectively. The $\mathrm{X}, \mathrm{Y}, \mathrm{Z}$ errors on GCPs, were within the accuracy specifications set. The pixel reprojection errors were above 1 pixel, which is a rule of thumb for relative orientation limits. Using very few GCPS, with spans of up to $3800 \mathrm{~m}$, it was suspected that the height accuracy would suffer, and an initial evaluation of the final DSM was done using Digital Terrain Model points from Department of Land and Syrveys of Cyprus. These points were measured in a $30 \times 30 \mathrm{~m}$ grid, from 1:25000 photo scale, digital photos. Although, the accuracy of the UAV flight should have been superior to DLS points due to photo scale, DLS photos were acquired by a photogrammetric camera, adjusted in a block, using GNSS control and check points to verify accuracy. The results revealed systematic differences of more than $2.5 \mathrm{~m}$ (Fig. 8) between the data sets, especially in the wide spans of the GCP, which can only be attributed to the poor adjustment of the UAV photos.

After the new field campaign for GCP collection, 163 were used in total for adjustment. The new adjustment reported 0.71 pixel reprojection error in tie points, 0.89 pixel reprojection error in GCPs and X, Y, Z errors of $0.10,0.08,0.19 \mathrm{~m}$ respectively. The $\mathrm{X}, \mathrm{Y}, \mathrm{Z}$ errors on GCP has increased, but remained within the EAC's specifications. EAC performed accuracy assessment of the delivered DSM, using 71 independent check points and reported Z RMS $0.35 \mathrm{~m}$, with max difference of $0.45 \mathrm{~m}$. These figures on the check points are consistent with the $Z$ error in GCP of $0.19 \mathrm{~m}$. Final products of orthophoto and DSM has resolution of $0.10 \mathrm{~m}$ and $0.50 \mathrm{~m}$ respectively.

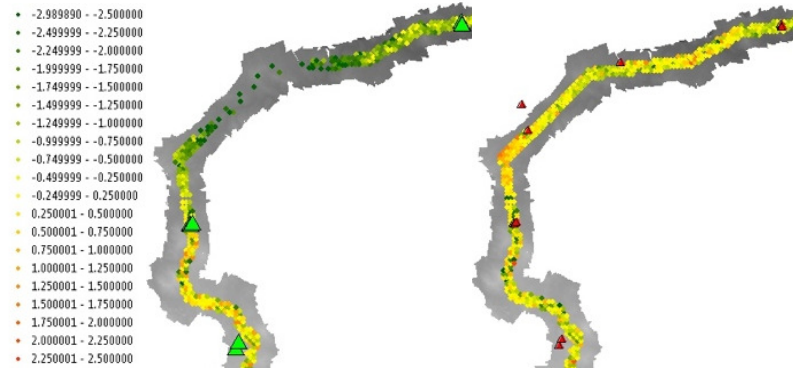

Figure 8. Checking of DSM before (left) and after (right) the addition of more GCPs 


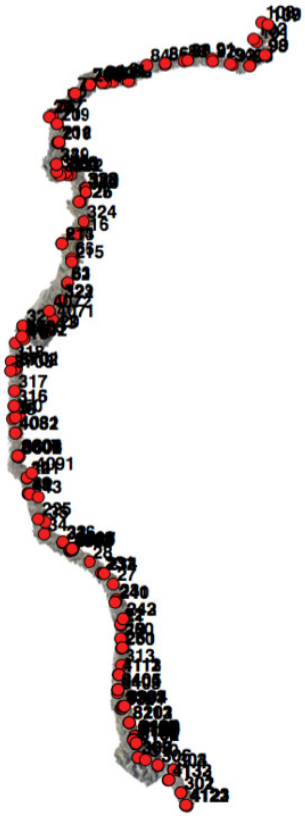

Figure 9. Final orthophoto with 163 GCP used

\section{CONCLUSSION AND DISCUSSION}

Extensive experience was gained thought this large project, and can be categorized in three aspects; UAV flights, data processing and cost/benefit analysis. Having been allowed to use a longer-range UAV, to cover the area in less flights, the task would have been significantly much easier and cost effective. At the same time, shadow differences would have been minimized from flight to flight, hence a visual pleasing orthophoto would have been attained.

Flight planning must accommodate terrain altitude, so that fixed scale is kept throughout the flight. If this is not accommodated by the UAV system, then large overlaps must be used and a lower and higher flight pass could provide adequate overlaps. This can be accomplished in a single flight, where the UAV can use the preferred flight height and on the way back it can fly at $120 \%$ of the preferred flight height. This kind of scale difference will not pose a problem for the processing software, while ensuring a larger corridor width and be helpful during the adjustment phase.

What seems to be a major concern in corridor surveys in mountainous areas is the unavoidable GCPs collection. The GCPs demand in corridor surveys, especially when using uncalibrated cameras, increase drastically. At this example, with photo coverage of $240 \mathrm{~m} \times 180 \mathrm{~m}$, control points were collected every $325 \mathrm{~m}$, or one every two photo widths. Use of automated processes, significant reduces the manual bulk of trivial tasks, but leads to overconfidence. Blunders must be removed prior to final optimization and realistic values should be used to retrieve faithful accuracy measures.

Given the flights regulations and the unfavorable terrain, the field campaign for data acquisition lasted 14 days. Data processing lasted 4 days, hence 18 days in total for the DSM and orthophoto. Alternatively, if traditional ground survey with GNSS took place, it would have lasted 14 days in the field and 3 days processing, 17 days in total for a vector plot and sparse contours. It should be mentioned that the final products are not directly comparable, and if a larger UAV was allowed, the overall workload would have been less for the UAV approach. Additionally, the cost for a light aircraft stationed in Greece, to perform aerial data acquisition using high a Nikon D800, was almost 20000€, without including the cost of GCP and photogrammetric processing.

Aerial corridor survey is a challenge task as it is, and using UAV adds peculiarities in all stages of it. Nevertheless, the project was successful, and UAV could provide a valid alternative in remote areas.

Since the project was completed, Cyprus has voted UAV regulations. These regulations will lead to UAV flight cost increase, but should still remain below the actual cost of hiring a manned aircraft for data acquisition, at least for areas up to 10 square kilometers.

\section{ACKNOWLEDGEMENTS}

The authors would like to acknowledge the Electricity authority of Cyprus for funding the HawkEye research project. The authors are particularly grateful to Mr. George Stavrou who supported the use of UAV for corridor mapping and Mr. Marios Miltonos for his decisive help in the field. The authors would also like to thank the department of Civil Aviation, for providing the permission for flying over the designated area and Department of Land and Surveys, for providing verification data for the DSM.

\section{REFERENCES}

Ameri, B., Meger, D., Power, K. and Gao, Y., 2008. UAS applications: disaster and emergency management. Proceedings of America Society of Photogrammetry and Remote Sensing Annual Conference, Baltimore, MD.

Eisenbeiß, H., 2009. UAV Photogrammetry. Dissertation, Institute of Geodesy and Photogrammetry, ETH Zurich, Switzerland.

Heintz, F., Rudol, P. and Doherty, P., 2007. From images to traffic behavior - a UAV tracking and monitoring application. IEEE International Conference on Information Fusion, pp. 1-8.

Kontitsis, M., Tsourveloudis, N. and Valavanis, K.P., 2004. A UAV vision system for airborne surveillance. IEEE International Conference on Robotics and Automation, New Orleans, LA, 1, pp. 77-83.

Kyratzis, A., Skarlatos, D., Menexes, G., Vamvakousis, V. and Katsiotis, A. 2017. Assessment of Vegetation Indices Derived by UAV Imagery for Durum Wheat Phenotyping Under a Water Limited and Heat Stressed Mediterranean Environment. Journal of Frontiers in Plant Science, section Crop Science and Horticulture, 8, Article 1114.

Rango, A., Laliberte, A., Steele, C., Herrick, J.E., Bestelmeyer, B., Schmugge, T., Roanhorse, A. and Jenkins, V., 2006. Using unmanned aerial vehicles for rangelands: current applications and future potentials. Environ. Pract., 8(3), pp. 159-168.

Remondino, F., Barazzetti, L., Nex, F., Scaioni, M. and Sarazzi, D., 2011. UAV photogrammetry for mapping and 3D modeling - current status and future perspectives. The International Archives of the Photogrammetry, Remote Sensing and Spatial Information Sciences, 38(1/C22). 
Saari, H., Antila, T., Holmlund, C., Mäkynen, J., Ojala, K., Toivanen, H., Pellikka, I., Tuominen, S., Pesonen, L. and Heikkilä, J., 2011. Unmanned Aerial Vehicle (UAV) operated spectral camera system for forest and agriculture applications. Proceedings of the SPIE 8174, Remote Sensing for Agriculture, Ecosystems, and Hydrology XIII, 19, p. 81740H.

Semsch, E., Jakob, M., Pavlicek, D. and Pechoucek, M., 2009. Autonomous UAV surveillance in complex urban environments. IEEE/WIC/ACM International Joint Conferences on Web Intelligence and Intelligent Agent Technologies, 2, WIIAT, pp. 82-85.

Skarlatos, D., Kiparissi, S. and Theodoridou, S., 2013. Direct Orthophoto Generation from Color Point Clouds of Complex Scenes. The International Archives of the Photogrammetry, Remote Sensing and Spatial Information Sciences, 40(1/W2), pp 367-371.
Skarlatos, D., Vlachos, M. and Vamvakousis, V., 2015. Investigating influence of UAV flight patterns in multi-stereo view DSM accuracy. Proc. SPIE 9528, Videometrics, Range Imaging, and Applications XIII, p. 95280M.

Srinivasan, S., Latchman, H., Shea, J., Wong, T. and McNair, J., 2004. Airborne traffic surveillance systems: video surveillance of highway traffic. Proceedings of the ACM 2nd international workshop on Video surveillance \& sensor networks, pp. 131-135.

Zhang, C. and Elaksher, A., 2011. An unmanned aerial vehicle based imaging system for 3D measurement of unpaved road surface distresses. J. Comput. Aided Civ. Infrastruct. Eng., 27(2), pp. 118-129. 